\title{
Stimulating Anopheles gambiae swarms in the laboratory: application for behavioural and fitness studies
}

Luca Facchinelli ${ }^{*} \mathbb{0}$, Laura Valerio ${ }^{1}$, Rosemary $S$ Lees $^{1}$, Clelia F Oliva ${ }^{1}$, Tania Persampieri ${ }^{1}$, C Matilda Collins $^{2}$, Andrea Crisanti ${ }^{1,3}$, Roberta Spaccapelo ${ }^{1}$ and Mark Q Benedict ${ }^{1,4}$

\begin{abstract}
Background: Male Anopheles mosquitoes that swarm rely in part on features of the environment including visual stimuli to locate swarms. Swarming is believed to be the primary behaviour during which mating occurs in the field, but is not a common behaviour in the laboratory. Features that stimulate male Anopheles gambiae G3 strain swarming were created in novel large indoor cages.

Methods: The following visual features were tested in all combinations to determine which were important for swarm formation. Large cages and fading ceiling lights at dusk alone did not stimulate swarming while a dark foreground and contrasting illuminated background with a contrasting landmark stimulated and localized swarm formation during artificial twilight. Given the need to test transgenic strains in as natural a setting as possible, in this study it was investigated whether induced swarm behaviour and cage size would affect relative mating performance of wild-type and transgenic $\beta 2 \mathrm{Ppo} 1$ and $\beta 2 \mathrm{Ppo} 2 \mathrm{~A}$. gambiae sexually sterile males.

Results: Even using a mosquito colony that has been in laboratory culture for 39 years, swarming behaviour was induced by this novel arrangement. The presence of swarming stimuli was associated with an increase in insemination frequency from 74.3 to $97.7 \%$ in large cages. Transgenic males showed a lower competitiveness in large cages compared to small cages regardless of the presence of swarming stimuli.

Conclusions: The results of the present study are discussed in view of the progressive evaluation of genetically modified A. gambiae strains and the potential applications of reproducing swarms in controlled conditions to dissect the mating behaviour of this species and the mechanisms controlling it.
\end{abstract}

Keywords: Anopheles gambiae, Swarms, Mating behaviour, Genetic control, Competitiveness

\section{Background}

Swarming by males is a natural mating behaviour of many Diptera [1, 2], including a major malaria vector mosquito, Anopheles gambiae sensu lato (s.l.) [3]. Swarms in Diptera are associated with overhanging trees, shrubs and contrasting-value ground markers [2], and among mosquitoes, swarms have been observed to occur during morning and evening twilight [1]. Anopheles gambiae

\footnotetext{
*Correspondence: lucafacchinelli@yahoo.it

${ }^{1}$ Department of Experimental Medicine, University of Perugia, Perugia, Italy

Full list of author information is available at the end of the article
}

swarms have been studied in the field, mainly in West Africa [4]. Males of this species specifically swarm near contrasting-shade ground features at evening twilight [4, 5], an activity lasting for approximately $30 \mathrm{~min}$. Swarms are composed almost entirely of males into which females briefly fly and leave the swarm in copula [6]. Copulation lasts less than $20 \mathrm{~s}$ [6]. Swarms can contain up to thousands of males though tens to several hundred is more common $[4,7]$. Field observations have produced the largest part of knowledge on mating behaviour of this species because $A$. gambiae swarms are difficult to obtain both in the laboratory and in large field cages [8], and such field work requires a great deal of experienced 
labour and long time periods to collect substantial data. The ability to reliably observe swarming in a controlled environment would thus be extremely valuable.

Mosquito insectaries typically provide light and dark periods to entrain behaviours including pupation, oviposition and mating, however wild anopheline mosquitoes brought into the laboratory do not mate at high rates making colonization difficult [9]. During the A. gambiae colonization process, environmental pressure acts on mating behaviour selecting stenogamy (in which mating takes place in restricted spaces like small cages) versus eurygamy (in which mating occurs in large spaces) [10]. Typical small laboratory cages (e.g. $30 \mathrm{~cm}$ per side) are not sufficiently large to permit swarming flights. There are few publications describing A. gambiae swarms indoors: Charlwood and Jones stimulated male swarming in a $1.7 \mathrm{~m}^{3}$ cage [11]; Marchand determined that provision of an artificial horizon that contrasted with a bright artificial sky encouraged swarming of A. gambiae and Anopheles arabiensis in a $<1 \mathrm{~m}^{3}$ cage [9]. Others [12-15] developed a large cage called a "mesocosm" where they studied effects of sugar on mating performance of A. gambiae. In their setting, A. gambiae males were observed to swarm but those studies did not focus on swarm behaviour nor on the stimuli inducing it. Clear evidence of swarming activity effects on female insemination rates has not been published.

Although A. gambiae is one of the most studied mosquito species worldwide due to its role in malaria transmission, its mating behaviour and the mechanisms regulating it remain unclear. Little information is available on the male-female-environment interactions during mating $[3,4,7,16]$ and little is known of the molecular mechanisms that regulate these processes $[17,18]$.

Efforts to develop genetically-modified mosquitoes (GMM) aimed at reducing malaria transmission will benefit from understanding mating interactions and factors that are associated with mating competitiveness. Mating behaviour represents a key component for the transmission of selected transgenes to natural populations, and poor mating competitiveness or altered reproductive behaviour of GMM resulting from the rearing history or the transgene could cause genetic control strategies to fail [19]. Part of the reason for these gaps in knowledge of $A$. gambiae biology is that swarms are difficult to study in natural settings. Locating them can be challenging, and it is difficult to gather quantitative measurements. It is almost impossible to design, run and replicate experiments on A. gambiae swarms in the field.

In this study, venues designed to stimulate more natural A. gambiae mating behaviour in order to improve behavioural studies and evaluate the mating performances of transgenic males in the laboratory were created. Six large insectary cages $\left(15.9 \mathrm{~m}^{3}\right.$ each) were designed, modifying and expanding on the visual stimuli described by Marchand [9], to consistently induce swarming activity in $A$. gambiae males. Swarms were observed and their effect on the female insemination rate and male competitiveness of two transgenic male-sterile $A$. gambiae lines [20] was measured. For genetic control strategies that require male releases into the environment, mating competitiveness is critical for success. The goal of this study was to optimize the evaluation of the mating performance of transgenic males in the laboratory and to develop a laboratory experimental setting suitable to study mating behaviour of this species.

The results obtained are discussed in the perspective of the progressive evaluation of genetically modified $A$. gambiae lines. The ground-breaking research that could be produced by investigating male swarming behaviour in enclosed environments with the goal of expanding the basic knowledge of $A$. gambiae mating biology is also discussed.

\section{Methods \\ Mosquito strains}

The Anopheles gambiae G3 strain [21] was employed for swarm observations, while mating studies were performed with A. gambiae G3 vs two A. gambiae sterile male $\beta 2$ Ppo lines [20]. G3 was chosen because it is widely used as wild type strain to create genetically engineered mosquitoes, while the transgenic males employed belong to $\beta 2$ Ppo lines that are maintained by continuously crossing transgenic females to G3 males [20]. This produces pools of genetically similar transgenic individuals and non-transgenic "wild-type" comparators every generation that were competed for virgin female mates with either of two strains of transgenic males: $\beta 2 \mathrm{Ppo} 1$ and $\beta 2 \mathrm{Ppo} 2$ [20]. In heterozygous males of the $\beta 2$ Ppo lines, the expression of the I-PpoI enzyme in testes induces a strong bias toward Y chromosome-carrying spermatozoa and complete early dominant embryo lethality in crosses with wild-type females [20]. In both strains, I-PpoI is fused to green fluorescent protein (GFP), both of which are expressed in sperm.

\section{Cage design and environmental conditions}

The study was carried out in the insectaries of the Department of Experimental Medicine, Functional Genomics Center of the University of Perugia. A total of six experimental cages were built in two large chambers, i.e. Insectary Field 1 measuring $6.68 \times 3.80 \times 3.00 \mathrm{~m}$ (all dimensions are $\mathrm{L} \times \mathrm{W} \times \mathrm{H}$ ), and Insectary Field 2 measuring $8.45 \times 3.80 \times 3.00 \mathrm{~m}$ (Figure 1). These studies were carried out in three types of cages: (1) $30 \mathrm{~cm}$ on each side 


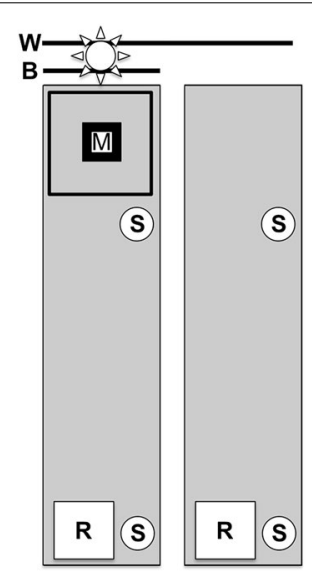

a

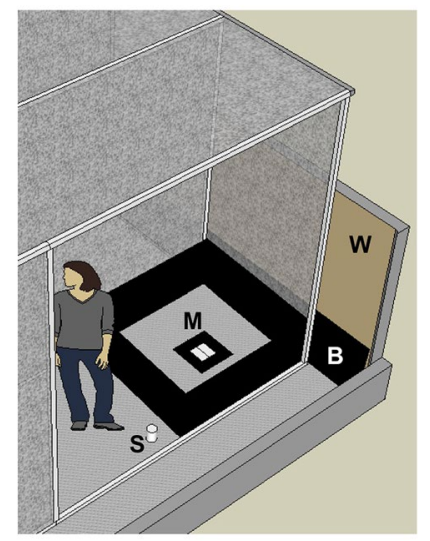

b
Figure 1 Illustration of the large cages. The typical arrangement of the swarming stimuli (left) is represented by the black-bordered swarm marker $(M)$ surrounded by the black artificial horizon lining the interior of each cage. Above these and outside of the cage, the black baffle $(B)$ is represented, preventing the three lights from shining directly into the cages but upward onto the wall $(W)$. Also shown are the locations of sugar sources $(S)$ and resting shelters $(R)$ flat against the floor. The swarming stimuli and controls were switched between rooms to determine whether there was any effect of insectary.

white plastic Bugdorms (BioQuip Products, Inc., Rancho Dominguez, CA, US); (2) custom-made large cages without swarming stimuli; (3) custom-made large cages with swarming stimuli. Large cages $(5.00 \times 1.22 \times 2.60 \mathrm{~m}$ each, Figure 1) consisted of white-painted wooden frames with walls and ceiling made of polyester mesh $(1,290 \mu \mathrm{m}$ openings, US size 15). Part of the front panel of each cage was made of a $50 \times 125 \mathrm{~cm}$ Plexiglas sheet provided with two $20 \mathrm{~cm}$ square entrances that allowed access to cages through affixed sleeves opening to the chamber. Each large cage was provided with a terracotta resting shelter which was kept humid and two $500 \mathrm{ml}$ cups containing a $10 \%$ sucrose solution, $0.1 \%$ methylparaben as preservative [22] and approximately $4 \mathrm{ml}$ acacia honey as an attractant. White absorbent paper placed in the cup allowed mosquitoes to land and get the sugar meal without drowning. In each large cage, swarming stimuli (Figure 1), consisted of (1) square arena made of four black plastic sheets (Correx equivalent, $122 \mathrm{~cm}$ long, $50 \mathrm{~cm}$ high and $0.5 \mathrm{~cm}$ thick), located at the back of the cages; (ii) a contrasting ground mark consisting of a black plastic square $(40 \times 40 \mathrm{~cm})$ and white plastic square $(20 \times 20 \mathrm{~cm})$ centred on top of it in the centre of the arena; (3) a $2.700 \mathrm{~K} 8 \mathrm{~W}$ compact fluorescent light located on the floor at the back of the cage hidden from the mosquitoes by a black plastic Correx equivalent sheet (122 cm long, $50 \mathrm{~cm}$ high and $0.5 \mathrm{~cm}$ thick), diffusing light onto the back wall of the chamber simulating twilight (horizon lights). Temperature and relative humidity were kept stable during the studies i.e. $28.01^{\circ} \mathrm{C}$ $\mathrm{SD} \pm 0.57^{\circ} \mathrm{C}$ and $72.1 \% \mathrm{SD} \pm 2.0 \%$.

Light in the chambers was provided by four or five ceiling light fixtures in Insectary Field 1 and Insectary Field 2, respectively, producing a diffused and evenly distributed light. Each light fixture was equipped with three T5 $80 \mathrm{~W}$ linear fluorescent bulbs with different emissions: 6.400, 4.000 and $2.700 \mathrm{~K}$. Emitting temperature, day duration, dusk and dawn fading of ceiling lights, were controlled by Easy Color Control software (OSRAM SpA, Società Riunite OSRAM Edison Clerici, Milan, Italy).

Length of daytime was the same for the two large cage treatments but light locations and durations differed. In both treatments, dawn lasted for $30 \mathrm{~m}$ from dark to full light, and full light lasted for 11:30 h. In the chamber where cages lacked the swarming stimuli, sunset lasted for $1 \mathrm{~h}: 30 \mathrm{~m}$ of fading ceiling from full light to minimum emission and $30 \mathrm{~m}$ of $2.700 \mathrm{~K}$ tubes only at minimum emission. In the chamber where cages where provided with swarming stimuli, sunset lasted for $1 \mathrm{~h}: 30 \mathrm{~m}$ of fading ceiling light from full light to minimum power, overlapping with $60 \mathrm{~m}$ of twilight provided by the horizon light. Different combinations of visual stimuli described above were tested, and the presence/absence of swarming males was detected using binoculars $(7 \times 35 \mathrm{~mm}$ roof prisms Foton, CCCP).

\section{Design of competitive mating experiment}

Assessing mating characteristics directly was facilitated by the fact that both strains have fluorescent sperm [20], which allowed us to determine whether wild-type, transgenic or neither type of male had mated females by examining their spermathecae. Because it is difficult to identify mixtures of fluorescent and wild-type sperm (the presence of fluorescent sperm is more easily concluded than mixtures), observations may bias the estimated frequency of matings in favour of transgenics. Only rarely (3 of 1,396 spermathecae) were we able to detect what appeared to be mixed matings. These cases were not included in the estimates of mating frequency. Larvae were reared using a diet reported previously [23]. Larvae were cultured according to a standard operating procedure (LV, personal communication). Two-day old virgin adults were introduced into three different type of cages: (1) Bugdorms; (2) large cages with visual stimuli inducing males to swarm; (3) large cages without visual stimuli. The mating competitiveness of the GMM lines was evaluated by introducing $25 \beta 2 \mathrm{Ppo} 1$ or $\beta 2 \mathrm{Ppo} 2$ males, $25 \mathrm{G} 3$ males, and 50 virgin $\mathrm{G} 3$ females in Bugdorms and $42 \mathrm{G} 3$ males, 42 $\beta 2$ Ppo males and 84 G3 females in large cages. 
For details of the study results, see Table 1. Adults were collected from the cages after a week, females were dissected and spermathecae examined for the presence of sperm and fluorescence. Competitive mating with $\beta 2 \mathrm{Ppo} 1$ line was replicated by reversing the treatments in either chamber in order to determine whether there was any effect of environmental differences.

\section{Ethics statement}

Adult females were fed using the Hemotek membrane feeding system (Discovery Workshops, Lancashire, England) and sterile cow blood (Allevamento Blood di Fiastra Maddalena, Teramo, Italy). The facility where the experiments were performed obtained the permit to host Class II genetically engineered organisms from the Italian Ministry of Health (permit no. PG/IC/OP2/13-002).

\section{Statistical analysis}

All analyses were performed using R 3.0.1. In all cases, the data are proportions arising from counts of two conditions: (a) those females that mated and those that did not, (b) the number of females mated by wild-type and the number of females mated by GMM males, and (c) the number of females, wild-type males and GMM males at the start of the experiment and alive at the end. Each combination was bound as a single response variable enabling their assessment by factorial analysis of deviance. There was overdispersion in all data and a quasibinomial model fit was used. Non-significant model terms were eliminated, starting with which insectary was used and proceeding thereafter by stepwise deletion; deletion tests used " $F$ " as appropriate to the overdispersion. Neither the date the experiment took place nor the individual

Table 1 Summary of competitive mating experiments employing males of $\beta 2 P p o 1$ line (above) and males of $\beta 2 P p o 2$ line (below)

\begin{tabular}{|c|c|c|c|c|c|c|c|c|}
\hline Cage & Treatment & $\begin{array}{l}\text { \# of adults per cage } \\
\beta 2 \mathrm{Ppo}^{\star} \text { ठ }^{-}: \mathrm{G}^{\star} 0^{\star} 0^{*}: \mathrm{G} 3 \%\end{array}$ & $\begin{array}{l}\text { (\%) G3 ô } \\
\text { survival }\end{array}$ & $\begin{array}{l}\text { (\%) } \beta 2 P p o 1 ઠ ð \\
\text { survival }\end{array}$ & (\%) $९$ survival & $\begin{array}{l}\text { Insemination } \\
\text { rate }\end{array}$ & $\begin{array}{l}\text { (\%) of }+\circ \\
\text { inseminated } \\
\text { by G3 } 0^{+} 0\end{array}$ & $\begin{array}{l}\text { (\%) of }+\circ \\
\text { inseminated } \\
\text { by } \beta 2 \mathrm{Ppo} 10^{+} 0^{+}\end{array}$ \\
\hline 1 & \multirow[t]{3}{*}{ Bugdorm } & \multirow[t]{3}{*}{$25: 25: 50$} & 53.6 & 46.4 & 76.0 & 73.7 & 75.0 & 25.0 \\
\hline 2 & & & 50.0 & 50.0 & 86.0 & 60.5 & 69.2 & 30.8 \\
\hline 3 & & & 54.1 & 45.9 & 82.0 & 75.6 & 71.0 & 29.0 \\
\hline A & \multirow[t]{3}{*}{ Large Rep 1} & \multirow[t]{3}{*}{$42: 42: 84$} & - & - & 54.8 & 65.2 & 96.7 & 3.3 \\
\hline B & & & - & - & 69.0 & 75.9 & 95.5 & 4.5 \\
\hline C & & & - & - & 72.6 & 76.7 & 97.8 & 4.3 \\
\hline D & \multirow{3}{*}{$\begin{array}{l}\text { Large swarm } \\
\text { Rep } 1\end{array}$} & \multirow[t]{3}{*}{$42: 42: 84$} & - & - & 71.4 & 98.3 & 93.2 & 6.8 \\
\hline$E$ & & & - & - & 82.1 & 100.0 & 85.1 & 14.9 \\
\hline $\mathrm{F}$ & & & - & - & 66.7 & 100.0 & 92.9 & 7.1 \\
\hline A & \multirow{3}{*}{$\begin{array}{l}\text { Large swarm } \\
\quad \text { Rep } 2\end{array}$} & \multirow[t]{3}{*}{$42: 42: 84$} & 59.5 & 50.0 & 82.1 & 97.1 & 94.0 & 7.5 \\
\hline B & & & 60.0 & 40.0 & 76.2 & 96.9 & 95.2 & 4.8 \\
\hline C & & & 52.4 & 11.9 & 63.1 & 94.3 & 94.0 & 6.0 \\
\hline D & \multirow[t]{3}{*}{ Large Rep 2} & \multirow[t]{3}{*}{$42: 42: 84$} & 78.6 & 50.0 & 78.6 & 74.2 & 81.6 & 18.4 \\
\hline$E$ & & & 71.4 & 11.9 & 77.4 & 61.5 & 97.5 & 2.5 \\
\hline$F$ & & & 61.9 & 35.7 & 73.8 & 83.3 & 89.8 & 10.2 \\
\hline Cage & Treatment & 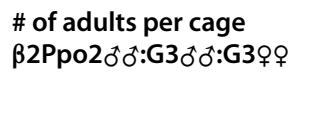 & $\begin{array}{l}\text { (\%) G3 } \text { ô }^{+} \\
\text {survival }\end{array}$ & $\begin{array}{l}\text { (\%) } \beta 2 \mathrm{Ppo}^{\star}{ }^{\star} \\
\text { survival }\end{array}$ & (\%) $९$ survival & $\begin{array}{l}\text { Insemination } \\
\text { rate }\end{array}$ & $\begin{array}{l}\text { (\%) of } \text { oᄋ } \\
\text { inseminated } \\
\text { by G3 } 0^{+}\end{array}$ & $\begin{array}{l}\text { (\%) of } q 9 \\
\text { inseminated } \\
\text { by } \beta 2 \mathrm{Ppo} 20^{\star}{ }^{\star}\end{array}$ \\
\hline 1 & Bugdorm & $25: 25: 50$ & 46.9 & 53.1 & 88.4 & 81.6 & 45.2 & 54.8 \\
\hline 2 & & & 52.4 & 47.6 & 90.0 & 86.7 & 69.2 & 30.8 \\
\hline 3 & & & 48.7 & 51.3 & 94.0 & 74.5 & 54.3 & 45.7 \\
\hline A & Large & $42: 42: 84$ & 85.7 & 59.5 & 72.6 & 83.6 & 84.3 & 15.7 \\
\hline B & & & 59.5 & 9.5 & 78.6 & 75.8 & 74.0 & 26.0 \\
\hline C & & & 35.7 & 40.5 & 70.2 & 72.9 & 65.1 & 34.9 \\
\hline D & Large swarm & $42: 42: 84$ & 66.6 & 28.6 & 76.2 & 98.4 & 82.5 & 17.5 \\
\hline$E$ & & & 47.6 & 23.8 & 86.9 & 97.3 & 77.5 & 22.5 \\
\hline$F$ & & & 57.1 & 14.3 & 70.2 & 96.6 & 82.5 & 17.5 \\
\hline
\end{tabular}

Cage number, treatment, number of adults introduced in each cage, WT and transgenic male survival, female survival, female insemination rate, and percentage of females inseminated by $G 3$ and $\beta 2$ Ppo males are shown. 
cage code were included in the final analyses, but were checked for influence and excluded a priori. Male survival in Replicate 1 was not recorded.

\section{Results and discussion}

\section{Stimulation of swarming behaviour}

Matings were measured in two sizes of cages: three "small cages" in which no swarm stimuli were present and six custom "large cages" in which swarm stimuli were either present or absent. Mosquitoes in large cages were observed to swarm only when four visual stimuli were present and which were used for all "swarm stimuli" treatments (Figure 1): (1) fading ceiling lights that simulate dusk; (2) $50 \mathrm{~cm}$ high black panels arranged as a square box-like open-top arena (1.22 $\mathrm{m}$ on each side), to create a $360^{\circ}$ artificial horizon; (3) a black and white landmark on the floor in the centre of the black arena; (4) an $8 \mathrm{~W}$ light located below the artificial horizon on one side of the cage (a "horizon light") shining upward on the lightcoloured wall to simulate a twilight sky. The last cue was important to stabilize the swarm above the landmark and to avoid males' phototactic activity toward the ceiling lights which was observed in preliminary trials. In all respects, the swarms appeared similar to what is observed naturally. In the large cages, swarms of $A$. gambiae males were obtained with their centre positioned at the level of the horizon consistently above the landmark, only in the presence of all the visual stimuli described above. The percentage of swarming males of the total number of males present in the cages is variable ranging between 20 and $50 \%$. Swarming behaviour started about $15 \mathrm{~min}$ after the beginning of the sunset beginning with 2-3 males. After that, more males joined the swarm that reached its maximum size when only the horizon lights were on, $30 \mathrm{~min}$ after the beginning of the twilight. During that time, the swarms had a sub-spherical shape, with their top slightly skewed toward the horizon lights. Its maximum size is about $60 \mathrm{~cm}$ wide and $50 \mathrm{~cm}$ height. In preliminary tests, the size of the cages (up to $3.00 \times 2.50 \times 2.50 \mathrm{~cm}$, $\mathrm{L} \times \mathrm{W} \times \mathrm{H}$ ), and the size of the black arenas (up to $3.00 \times 2.50 \times 1.50 \mathrm{~cm}, \mathrm{~L} \times \mathrm{W} \times \mathrm{H}$ ) were increased. The swarm shape and size changed according to the cage setting maintaining almost the same width (about $60 \mathrm{~cm}$ ) but increasing in height up to $1.00 \mathrm{~m}$. In all cases, swarms were close to the cage floor with the lower males flying about $30 \mathrm{~cm}$ from the contrasting marker on the floor.

Routine laboratory culture selects strongly for stenogamy and it is surprising that selection has not eventually resulted in insemination of all females in small cages, particularly in a strain such as G3 that has been maintained in laboratories since 1975, approximately 730 generations. In preparatory tests, we used similar visual stimuli in small cages without obtaining an increase of mating frequency [67 and 58\%, respectively with (4 replicates) and without (5 replicates) swarming cues, G test for goodness of fit, $G=0.089, p=0.765]$. Combined results demonstrated that although an increase in male flight activity and several mating events were observed at dusk in small cages, the characteristic male "dancing" activity associated with swarming at dusk was not observed. Flight activity of large numbers of males toward ceiling lights during the sunset phase was also observed in large cages without swarming stimuli, due apparently to male phototactic response, but it could not be described as swarm behaviour and no increase in female insemination rates was observed.

Results of mating competition experiments are summarized in Table 1.

\section{Adult survival and effect of swarming on proportions of females mated}

Swarming is a behaviour that requires male flight for prolonged periods. Larger cages might demand greater flight performance of both males and females and affect adults' ability to find shelter and sugar. The effects of swarming and cage size on male and female survival (after 7 days of cohabitation in all cases) for a wild-type comparator (G3 strain) and two strains of transgenic males [20] were determined.

\section{G3 females}

Considering all cages, neither the environmental chamber used nor the strain of males with which females cohabited affected survival (Table 2). However, wild-type female survival after 7 days was significantly higher in small cages $(0.84 \pm 0.03)$ than in large ones $(0.76 \pm 0.02)$. These explanatory variables, however, capture only $26 \%$ of the deviance in female survival. In large cages in which an effect of swarming could be detected, female survival was not influenced by either the strain of transgenic males with which they were housed (Table 2), by swarming, nor by their interaction. These factors explain only $5 \%$ of the deviance.

\section{G3 males}

Overall, non-transgenic comparator males survived at a higher rate than transgenic males $(0.65 \pm 0.06$ vs $\left.0.45 \pm 0.06, \mathrm{~F}_{17,18}=27.1, \mathrm{p}<0.01\right)$. For wild type males, cage size, strain of transgenic male with which wild-type males were housed and environmental chamber captured $22 \%$ of the variance in survival considering all cages. Wild-type male survival did not vary as a function of strain with which they competed or cage size. In the large cages, the model accounted for $14 \%$ of the deviance in the data and was a poor fit. Wild-type male survival was not affected by swarming, the strain of transgenic male with which they were housed nor by their interaction. 
Table 2 Summary of the model deletion tests to identify effects on the survival of the Anopheles gambiae

\begin{tabular}{|c|c|c|c|c|c|c|c|c|c|}
\hline & \multicolumn{3}{|c|}{ Female survival } & \multicolumn{3}{|c|}{ Wild type male survival } & \multicolumn{3}{|c|}{ GMM male survival } \\
\hline & $F$ & d.f. & $p$ & $\mathbf{F}$ & d.f. & $\mathrm{p}$ & $\mathbf{F}$ & d.f. & $p$ \\
\hline \multicolumn{10}{|l|}{ All cages } \\
\hline Insectary & 0.05 & 14,15 & 0.82 & 1.37 & 14,15 & 0.26 & 0.67 & 14,15 & 0.43 \\
\hline Strain & 0.78 & 13,14 & 0.39 & 0.25 & 15,16 & 0.63 & 0.02 & 15,16 & 0.88 \\
\hline Cage Size & 4.72 & 16,17 & $<0.05$ & 2.56 & 16,17 & 0.13 & 15.81 & 16,17 & $<0.01$ \\
\hline \multicolumn{10}{|l|}{ Large cages only } \\
\hline Strain:Swarm & 0.18 & 8,9 & 0.68 & 0.25 & 8,9 & 0.63 & 0.67 & 8,9 & 0.44 \\
\hline Strain & 0.33 & 10,11 & 0.57 & 0.34 & 9,10 & 0.57 & 0.19 & 9,10 & 0.67 \\
\hline Swarm & 0.00 & 9,10 & 0.96 & 0.94 & 10,11 & 0.35 & 0.21 & 10,11 & 0.66 \\
\hline
\end{tabular}

Statistically significant results are in italics.

\section{Transgenic males}

The full model, which considered transgenic male survival in all cages, accounted for $52 \%$ of the deviance. No difference between the survival of transgenic strain males was detected but survival was greater for both strains in the smaller cages $(71 \pm 0.05 \%$ vs $32 \pm 0.04 \%)$ (Table 2$)$. Within the large cages, the model was a very poor fit and explained only $10 \%$ of the deviance in survival. Neither strain nor swarming contributed significantly to variation in survival nor was survival affected by an interaction between strain and swarming.

\section{Female insemination rate}

Only one explanatory variable affected the proportion of females mated and that was swarm venue presence in large cages (Figure 2). The proportion of matings was significantly higher in cages containing venues in which males had been observed to swarm $\left(\mathrm{F}_{16,17}=136.45\right.$, $\mathrm{p}<0.001)$. Neither strain of cohabiting transgenic male $\left(\mathrm{F}_{15,16}=0.59, \mathrm{p}=0.45\right)$ nor its interaction with swarming $\left(\mathrm{F}_{14,15}=0.28, \mathrm{p}=0.60\right)$ were significant contributors. Overall, neither the insectary, type of cohabiting male nor cage size affected the proportion of females mated (Table 3). The proportions of females mated did not differ depending on which transgenic strain of male was present nor was there an interaction between transgenic strain and swarm venue stimuli.

The observation of male-swarming correlating with higher mating frequencies draws attention to the male's role in mating and to the possible stimulation of female receptiveness by swarming activity. In Aedes aegypti, swarming males produce a volatile aggregation pheromone that attracts both males and females [24]. It is not known if the same happens in A. gambiae and these two species have a different swarming behaviour and mating strategy. The mechanism that results in difficulty in obtaining matings in the lab among field collected mosquitoes may be that many females will not mate except

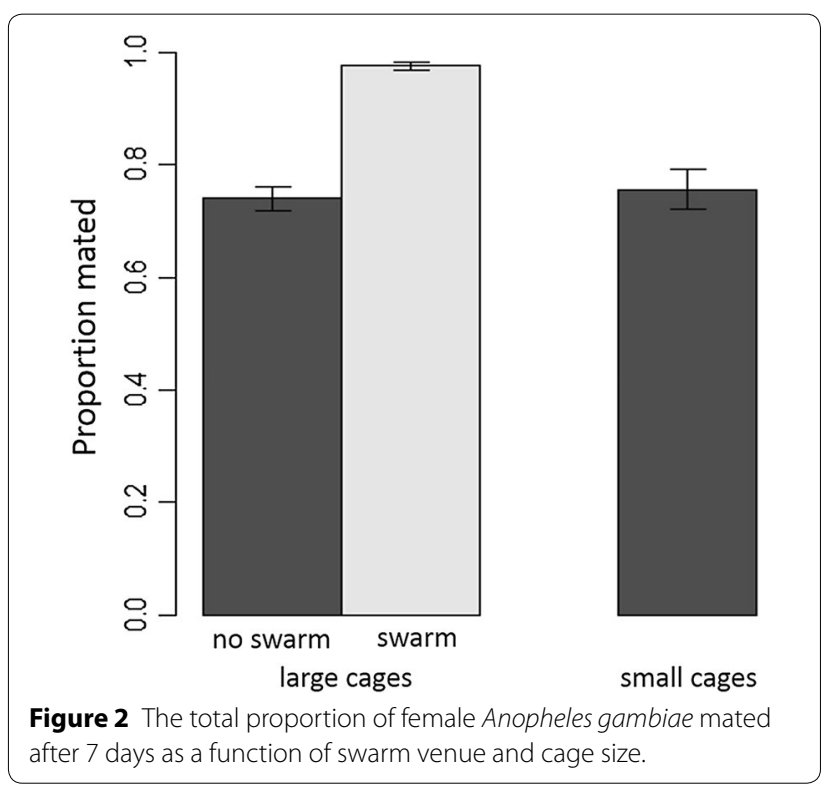

in a swarm. It remains difficult to know if male attempts at copulation are less frequent when they do not swarm or if some females are rejecting males that are attempting to copulate outside of a swarm formation. The propensity of at least a significant proportion of adults to mate in swarms has not been lost in these strains and we have shown in large cages that swarm behaviour increases insemination rate by approximately $24 \%$. These data are consistent with the results of Dao et al. [25], which showed that A. gambiae probably has an alternative mating strategy such that not all mating occurs in swarms. In the field, Dao et al. calculated that mating in resting places where males do not swarm occurs at a low rate (between 6 and 15\%), and it is plausible that this frequency increases during colonization in small cages, becoming predominant over mating in swarms after several generations in captivity, as detected in this study. 
Table 3 Summary of the model deletion tests to identify effects on the mated status of female Anopheles gambiae

\begin{tabular}{|c|c|c|c|c|c|c|}
\hline \multirow[t]{2}{*}{ Both cage sizes } & \multicolumn{3}{|c|}{ Proportion of females mated } & \multicolumn{3}{|c|}{ Proportion of transgenic matings } \\
\hline & $\mathrm{F}$ & d.f. & $p$ & $\mathrm{~F}$ & d.f. & $\mathrm{p}$ \\
\hline Insectary & 1.19 & 19,20 & 0.28 & 1.20 & 19,20 & 0.29 \\
\hline Strain & 3.46 & 21,22 & 0.08 & 27.44 & 21,22 & $<0.001$ \\
\hline Cage Size & 0.03 & 20,21 & 0.87 & 26.54 & 21,22 & $<0.001$ \\
\hline Swarm & 134.45 & 22,23 & $<0.001$ & 0.62 & 20,21 & 0.44 \\
\hline
\end{tabular}

Statistically significant results are in italics.

Female insemination rates obtained in large cages in other studies where swarms were observed [13, 14], were not as high as those we obtained. It is difficult to explain why but authors speculate that the visual stimuli produced, which were able to induce males to swarm, also have an effect on virgin females attracting them to the mating arena, a behaviour already reported [11].

\section{Competitiveness of transgenic males in the various settings}

Short-term mating competitiveness experiments assess an important component of fitness and conducting them in large cages provides important preliminary information on the performance of GMM [26]. Considering only large cages in this study, neither the insectary used nor swarm stimuli affected the proportion of matings by transgenic males in large cages (Figure 3, Table 3). Nor did the ratio of transgenic to non-transgenic males surviving at the end of the experiment have any effect on the proportion of transgenic matings $\left(\mathrm{F}_{15,16}=1.31\right.$, $\mathrm{p}=0.27$ ). In contrast, the two cage sizes had very different proportions of transgenic male matings: transgenic males competed more successfully in small cages vs. large ones. The strains also differed: $\beta 2 \mathrm{Ppo} 2$ achieved a higher proportion of matings than $\beta 2 \mathrm{Ppo} 1$ in all cases. The mating competitiveness estimates of " $\mathrm{C}$ ", based on the Fried

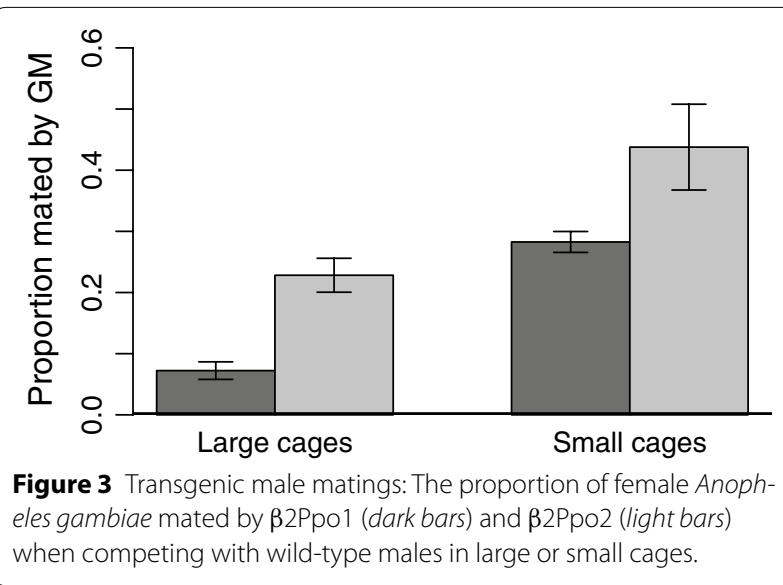

index [27], of male $\beta 2 \mathrm{Ppo} 1$ and $\beta 2 \mathrm{Ppo} 2$, respectively in small cages were $0.396( \pm 0.033)$ and $0.834( \pm 0.222)$ and $0.081( \pm 0.18)$ and $0.305( \pm 0.051)$ in large cages.

Unexpectedly, the proportion of females inseminated by transgenic males was not affected by transgenic male survival (Figure 4), being lower than that of non-transgenic males in large cages. This suggested that matings were completed early during the course of each experiment. In subsequent mating studies, it was observed that when large cages were populated with 2-day old virgin nontransgenic adults, 97.3 and $77.3 \%$ of females were inseminated in "swarm" and "no-swarm" treatments respectively, after only two nights. These values were similar after 5 and 7 nights in concurrent experiments. Therefore, $A$. gambiae male survival may not be a strong determinant of mating competitiveness in assays using cohorts of coetaneous adults. This finding should be taken into account for modelling purposes and allows mating competition experiments to be performed more quickly with A. gambiae, since they currently often take around 1 week [28]. The preparatory test described above indicate that most matings occur early during the course of the experiments and the insemination rate does not change over the subsequent 7 days. It has been shown that in different mosquito species, a single male can mate several females and males can replenish their sperm supply when depleted [29]. Therefore, it is reasonable to assume that the males that mated the majority of females within 2 days in the "no-swarm" treatments had a sufficient supply of sperm for mating the remainder within 7 days but did not. One might hypothesize that the mating limitation in the noswarm treatments depends upon a female behaviour that is not stimulated under these conditions.

Independent studies indicated that competitiveness of these transgenic strains is consistent with the hypothesis that larger cages challenge mating ability more than small ones. The same transgenic strains tested here were competed previously in intermediate sized cages of $8.5 \mathrm{~m}^{3}$ [28]. Those cages did not contain any features identified here that might stimulate swarming (MQB personal communication). They obtained a similar proportion of inseminated females (75\% after 7 days), as we observed 


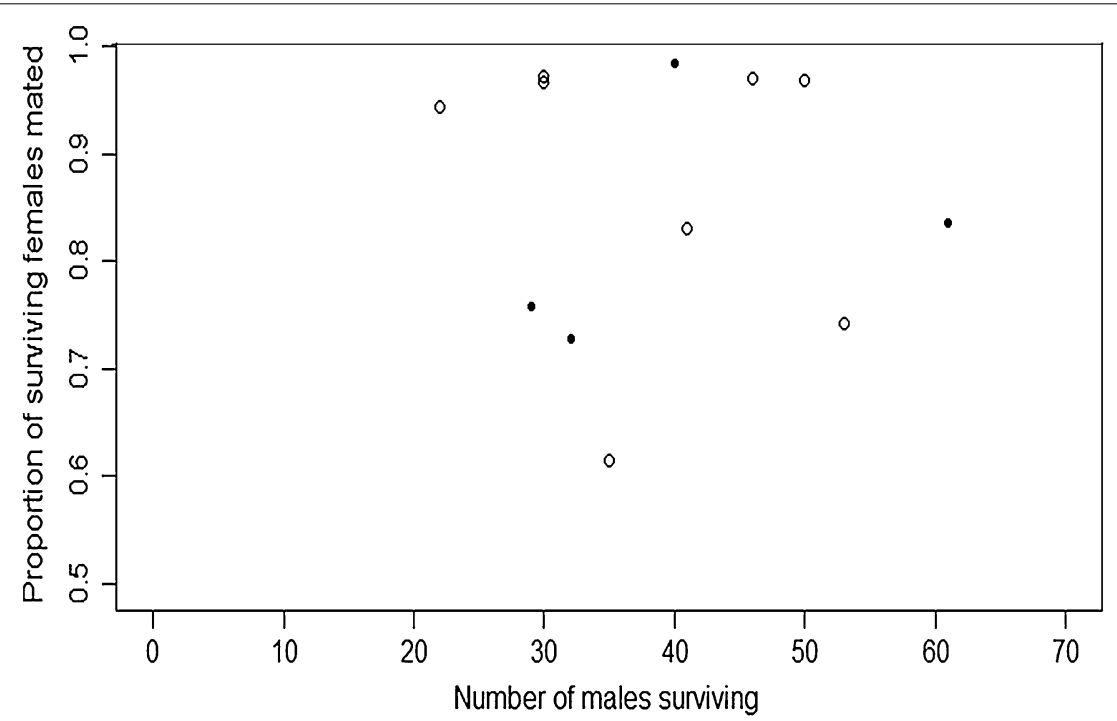

Figure 4 Proportion of inseminated females as a function of surviving males.

here in the absence of swarming. The authors observed estimates of competitiveness intermediate to those determined here in smaller and larger cages: $0.116( \pm 0.079)$ and 0.640 ( \pm 0.036$)$ for $\beta 2 \mathrm{Ppo} 1$ and $\beta 2 \mathrm{Ppo} 2$, respectively.

\section{Conclusions}

The ability to reliably stimulate swarming in the laboratory in large cages provides both insights and an opportunity to study questions related to factors controlling assortative mating, perception and the evaluation of strains being considered for field release. The lack of stimuli for swarm behaviour, where most natural mating is believed to occur, and culture in small cages, mean that $A$. gambiae mating behaviour in insectaries is quite different from that which occurs in the field. Results from studies aimed at revealing factors affecting mating behaviour might only be a poor proxy for natural behaviour if males are in laboratory cages and do not have the possibility to swarm.

These data together demonstrate that for these strains, males that are observed to be inferior when competing for mates in small cages can be expected to have even less ability to compete in larger cages.

Previous research on A. gambiae swarms in the laboratory [9-11] provided a basis to study the natural mating behaviour of this species in enclosed environments. Although several studies describing A. gambiae mating behaviour in the field have been published since then [3, $4,7,16,30,31]$, parallel research in the insectary has not been developed. The main goals of swarm studies in the field have been to explain mating choice and reproductive isolation within the A. gambiae complex. Incipient speciation between the $\mathrm{M}$ and $\mathrm{S}$ molecular forms, now elevated to species rank [32], has been discussed [33], and there is evidence showing that gene flow between the molecular forms is reduced due to the presence of premating barriers. Reproducing $\mathrm{M}$ and $\mathrm{S}$ swarm segregation in the laboratory might provide the basis for revealing the mechanisms controlling assortative mating amongst them and will be a major component of our future activities. The behavioural processes leading to this reproductive isolation among wild populations are still not fully understood and seem to vary among populations and geographical areas [34]. The lack of a model that reproduces swarm behaviour in laboratory cages makes it difficult to investigate the mechanisms controlling the reproductive isolation within the A. gambiae complex. A starting point to reveal the mechanisms regulating non-random matings between $\mathrm{M}$ and $\mathrm{S}$ in the open field is to reproduce and study their mating behaviour in large insectary cages.

Results presented here are critical for achieving a more realistic evaluation of the mating performance, and fitness in general, of the GMM A. gambiae lines. The use of similar methods to reproduce $A$. gambiae swarms in experiments aimed at assessing the fitness cost of genetic modifications and more generally to study different aspects of mating behaviour of this species are strongly encouraged.

\section{Authors' contributions}

LF and MQB designed the study and drafted the manuscript; LF, LV, RSL, TP, and CO collected data; CMC performed the statistical analysis, RS and AC contributed to data interpretation; all authors revised the manuscript critically for important intellectual content. All authors read and approved the final manuscript. 


\section{Author details}

${ }^{1}$ Department of Experimental Medicine, University of Perugia, Perugia, Italy. ${ }^{2}$ Centre for Environmental Policy, Imperial College London, London, UK. ${ }^{3}$ Department of Life Sciences, Imperial College London, London, UK. ${ }^{4}$ Present Address: Centers for Disease Control and Prevention, Atlanta, USA.

\section{Acknowledgements}

Funded by a Grant from the Foundation for the National Institutes of Health through the Vector-Based Control of Transmission Discovery Research (VCTR) program of the Grand Challenges in Global Health Initiative of the Bill \& Melinda Gates Foundation. Additional funding was received from the European Union's Seventh Framework Programme (FP7/2007-2013) under Grant agreement no 218164 and RSL and CO have received funding from the same programme under the Marie Curie Actions (Co-funding of Regional, National and International Programmes-COFUND) project I-MOVE grant agreement no 267232.

\section{Compliance with ethical guidelines}

\section{Competing interests}

The authors declare that they have no competing interests.

Received: 4 March 2015 Accepted: 3 July 2015

Published online: 15 July 2015

\section{References}

1. Yuval B (2006) Mating systems of blood feeding flies. Ann Rev Entomol 51:413-440

2. Jones JA (1969) The swarming and mating flight of Diptera. Ann Rev Entomol. 14:271-298

3. Charlwood JD, Pinto J, Sousa CA, Madsen H, Ferreira C, do Rosario VE (2002) The swarming and mating behaviour of Anopheles gambiae s.s. (Diptera: Culicidae) from São Tomé Island. J Vector Ecol. 27:178-183

4. Sawadogo PS, Namountougou M, Toé KH, Rouamba J, Maïga H, Ouédraogo KR et al (2014) Swarming behaviour in natural populations of Anopheles gambiae and An. coluzzii. Review of 4 years survey in rural areas of sympatry, Burkina Faso (West Africa). Acta Trop 132(Suppl):42-52

5. Howell PI, Knols BG (2009) Male mating biology. Malar J. 8(Suppl 2):8

6. Charlwood JD, Jones DR (1979) Mating behavior in the mosquito, Anopheles gambiae s.l. I. Close range and contact behavior. Physiol Entomol 5:305-320

7. Diabaté A, Yaro AS, Dao A, Diallo M, Huestis DL, Lehmann T (2011) Spatial distribution and male mating success of Anopheles gambiae swarms. BMC Evol Bio 11:184

8. Knols BGJ, Njiru BN, Mathenge EM, Mukabana RW, Beier JC, Killeen GF (2002) Malariasphere: a greenhouse-enclosed simulation of a natural Anopheles gambiae ecosystem. Malaria J. 1:1-19

9. Marchand RP (1985) A new cage for observing mating behavior of wild Anopheles gambiae in the laboratory. J Am Mosq Control Assoc. 1:234-236

10. Charlwood JD May the force be with you: measuring mosquito fitness in the field. In Ecological Aspects for Application of Genetically Modified Mosquitoes. In: Takken W, Scott TW; Wageningen UR (eds) Frontis Series Volume 2. Wageningen, pp 47-62. Available at: http://www.edepot.wur. $\mathrm{nl} / 136904$ Accessed Sep 2014

11. Charlwood JD, Jones MDR (1980) Mating in the mosquito, Anopheles gambiae s.l. II. Swarming behaviour. Physiol Entomol. 5:315-320

12. Stone CM, Taylor RM, Foster WA (2009) An effective indoor mesocosm for studying populations of Anopheles gambiae in temperate climates. J Am Mosq Control Assoc. 25:514-516

13. Stone CM, Taylor RM, Roitberg BD, Foster WA (2009) Sugar deprivation reduces insemination of Anopheles gambiae (Diptera: Culicidae), despite daily recruitment of adults, and predicts decline in model populations. J Med Entomol 46:1327-1337

14. Gary RE Jr, Cannon JW 3rd, Foster WA (2009) Effect of sugar on male Anopheles gambiae mating performance, as modified by temperature, space, and body size. Parasit Vectors. 22:2-19

15. Jackson BT, Stone CM, Ebrahimi B, Briët OJ, Foster WA (2015) A low-cost mesocosm for the study of behaviour and reproductive potential in Afrotropical mosquito (Diptera: Culicidae) vectors of malaria. Med Vet Entomol 29:104-109
16. Dabiré KR, Sawadodgo S, Diabaté A, Toe KH, Kengne P, Ouari A et al (2013) Assortative mating in mixed swarms of the mosquito Anopheles gambiae s.s. M and S molecular forms, in Burkina Faso, West Africa. Med Vet Entomol 273:298-312

17. Shaw WR, Teodori E, Mitchell SN, Baldini F, Gabrieli P, Rogers DW et al (2014) Mating activates the heme peroxidase HPX15 in the sperm storage organ to ensure fertility in Anopheles gambiae. Proc Natl Acad Sci 111:5854-5859

18. Thailayil J, Magnusson K, Godfray HC, Crisanti A, Catteruccia F (2011) Spermless males elicit large-scale female responses to mating in the malaria mosquito Anopheles gambiae. Proc Natl Acad Sci 108:13677-13681

19. Scott TW, Rasgon JL, Black WC, Gould F Fitness studies: developing a consensus methodology. In: Knols BG, Frontis LC (eds) Bridging laboratory and field research for genetic control of disease vectors. Wageningen UR Frontis Series, vol 11. The Netherlands, pp 171-181. Available at: http://www. library.wur.nl/frontis/disease_vectors/16_scott.pdf. Accessed Feb 2015

20. Windbichler N, Papathanos PA, Crisanti A (2008) Targeting the X chromosome during spermatogenesis induces $Y$ chromosome transmission ratio distortion and early dominant embryo lethality in Anopheles gambiae. PLoS Genet 4:e1000291. doi:10.1371/journal.pgen.1000291

21. MR4 wild stocks information. https://www.mr4.org/Portals/3/Pdfs/ Anopheles/G3\%20Stock\%20Auth\%20Sheet.pdf. Accessed February 2015

22. Benedict MQ, Hood-Nowotny RC, Howell PI, Wilkins EE (2009) Methylparaben in Anopheles gambiae s.l. sugar meals increases longevity and malaria oocyst abundance but is not a preferred diet. J Insect Physiol 55:197-204

23. Damiens D, Benedict MQ, Wille M, Gilles JR (2012) An inexpensive and effective larval diet for Anopheles arabiensis (Diptera: Culicidae): eat like a horse, a bird, or a fish? J Med Entomol 49:1001-1011

24. Cabrera M, Jaffe K (2007) An aggregation pheromone modulates lekking behavior in the vector mosquito Aedes aegypti (Diptera: Culicidae). J Am Mosq Control Assoc. 1:1-10

25. Dao A, Adamou A, Yaro AS, Maïga HM, Kassogue Y, Traoré SKF et al (2008) Assessment of alternative mating strategies in Anopheles gambiae: Does mating occur indoors? J Med Entomol 45:643-652

26. Facchinelli L, Valerio L, Ramsey JM, Gould F, Walsh RK, Bond G et al (2013) Field cage studies and progressive evaluation of genetically-engineered mosquitoes. PLoS Negl Trop Dis. 7(1):e2001. doi:10.1371/journal.pntd.0002001

27. Fried M (1971) Determination of sterile insect competitiveness. J Econ Entomol 64:869-872

28. Klein TA, Windbichler N, Deredec A, Burt A, Benedict MQ (2012) Infertility resulting from transgenic I-Ppol male Anopheles gambiae in large cage trials. Pathog Glob Health. 1:20-31

29. Mahmood F, Reisen WK (1982) Anopheles stephensi (Diptera: Culicidae): changes in male mating competence and reproductive system morphology associated with aging and mating. J Med Entomol 19:573-588

30. Sawadogo SP, Costantini C, Pennetier C, Diabaté A, Gibson G, Dabiré RK (2013) Differences in timing of mating swarms in sympatric populations of Anopheles coluzzii and Anopheles gambiae s.s. (formerly An. gambiae M and S molecular forms) in Burkina Faso, West Africa. Parasit Vectors. 6:275

31. Assogba BS, Djogbénou L, Saizonou J, Diabaté A, Dabiré RK, Moiroux N et al (2014) Characterization of swarming and mating behaviour between Anopheles coluzzii and Anopheles melas in a sympatry area of Benin. Acta Trop 132(Suppl):53-63

32. Coetzee M, Hunt RH, Wilkerson R, della Torre A, Coulibaly MB, Besansky N (2013) Anopheles coluzzii and Anopheles amharicus, new members of the Anopheles gambiae complex. Zootaxa. 3619:246-274

33. della Torre A, Costantini C, Besansky NJ, Caccone A, Petrarca V, Powell JR et al (2002) Speciation within Anopheles gambiae-the glass is half full. Science. 298:115-117

34. Lanzaro CG, Yoosook L (2013) Speciation in Anopheles gambiae - The distribution of genetic polymorphism and patterns of reproductive isolation among natural populations. In: Manguin S (ed) Anopheles mosquitoes-new insights into malaria vectors. InTech. Available at: http:// www.intechopen.com/books/anopheles-mosquitoes-new-insights-intomalaria-vectors/speciation-in-anopheles-gambiae-the-distribution-ofgenetic-polymorphism-and-patterns-of-reproductiv. Accessed February 2015 